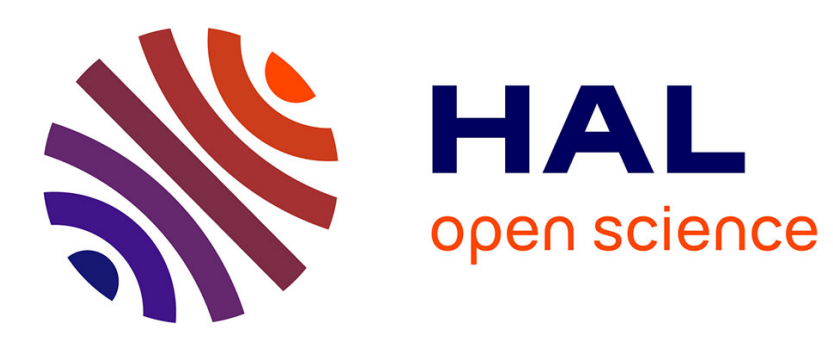

\title{
A Semiquantal Approximation to the Bound-state Coulomb Problem
}

\author{
R.K. Bhaduri, M. Brack, H. Gräf, P. Schuck
}

\section{To cite this version:}

R.K. Bhaduri, M. Brack, H. Gräf, P. Schuck. A Semiquantal Approximation to the Bound-state Coulomb Problem. Journal de Physique Lettres, 1980, 41 (15), pp.347-350. 10.1051/jphyslet:019800041015034700 . jpa-00231795

\section{HAL Id: jpa-00231795 https://hal.science/jpa-00231795}

Submitted on 1 Jan 1980

HAL is a multi-disciplinary open access archive for the deposit and dissemination of scientific research documents, whether they are published or not. The documents may come from teaching and research institutions in France or abroad, or from public or private research centers.
L'archive ouverte pluridisciplinaire HAL, est destinée au dépôt et à la diffusion de documents scientifiques de niveau recherche, publiés ou non, émanant des établissements d'enseignement et de recherche français ou étrangers, des laboratoires publics ou privés. 


\title{
A Semiquantal Approximation to the Bound-state Coulomb Problem
}

\author{
R. K. Bhaduri $\left({ }^{*}\right)$, M. Brack $\left({ }^{* *}\right)$, H. Gräf and P. Schuck \\ Institute Laue-Langevin, BP 156X, 38042 Grenoble, France
}

(Reçu le 29 avril 1980, accepté le 17 juin 1980)

\begin{abstract}
Résumé. - Nous utilisons une approche semi-quantique sans paramètre ajustable pour calculer la densité et l'énergie dans le problème de l'état de liaison Coulombien. Contrairement à la plupart des méthodes semi-classiques courantes notre approche n'utilise pas la fonctionnelle de la densité de l'énergie. L'application auto-consistante de la méthode au problème atomique est illustrée par un exemple numérique.
\end{abstract}

\begin{abstract}
A parameter-free semiquantal approach is used to calculate the density and energy in the boundstate Coulomb problem. Unlike most semiclassical methods in current use, our approach dispenses with the energy density functional. The self-consistent application of the method to the atomic problem is illustrated by a numerical example.
\end{abstract}

The Coulomb potential plays a central role in atomic and molecular physics. Even in the mean field approximation, quantum mechanical calculations require considerable computational effort, particularly for molecules. For this reason, much work has been done [1-8] using semiclassical methods which are based on extensions of the ThomasFermi (TF) approximation [9]. A basic difficulty in the simplest atomic TF model is the divergence of the spatial density at the centre, leading to a sizeable overestimation of the binding energy. This difficulty is present even when one ignores the electronelectron interaction. The divergence of the density is removed [1] in a variational calculation by adding the Weizsäcker correction to the kinetic energy density functional. However, in order to obtain a very good agreement of the energy with the corresponding quantum mechanical result, an adjusted coefficient of the Weizsäcker term is required [2,3]. This phenomenological energy functional has been used recently by Gross and Dreizler [8] for diatomic molecules.

In this work we adopt a different and perhaps more rigorous semiquantal approach within the mean field scheme, which is parameter-free and may be extended to the multicentre molecular problem.

(*) Permanent address : Dept. of Physics, McMaster University, Hamilton, Ontario, Canada.

(**) Permanent address : University of Regensburg, Institute for Theoretical Physics, Regensburg, W-Germany.
All quantum mechanical information are contained in the Bloch density matrix [10], from which the electronic ground state spatial and kinetic energy densities and the total energy may be obtained by suitable Laplace inversions. We make systematic approximations to the Bloch density that avoid the divergence at the origin, and then go on to calculate the bulk electronic properties. In this paper, to illustrate our method, we mainly concentrate on the model problem of $Z$ noninteracting electrons moving in the bare Coulomb field of the nucleus, as was done by Yonei and Tomishima [2]. The semiquantal solution of this problem is nontrivial. Its extension to the atomic self-consistent case by inclusion of the electron-electron interaction is straightforward. This is done at the end of the paper and a numerical example is included.

In our model problem, the wave functions $\psi_{i}$ and the eigenenergies $\varepsilon_{i}$ of the electrons are determined from the one-body Schrödinger equation with the nuclear potential $V_{\mathrm{N}}=-Z e^{2} / r$. It is easy to calculate analytically the spatial density

$$
\rho(r)=\sum_{i}^{\prime}\left|\psi_{i}(r)\right|^{2}
$$

and the energy $E=\sum_{i}^{\prime} \varepsilon_{i}$ of the $Z$ electrons, where $\sum_{i}^{\prime}$ denotes summation over the occupied states. Our aim is to develop a semiquantal method which will enable us to calculate $E$ and $\rho$ directly from the potential, with no need to solve for the wave functions. 
The single particle Bloch density matrix is defined by the equation

$$
\begin{aligned}
C\left(\mathbf{r}, \mathbf{r}^{\prime}, \beta\right)=\sum_{i} \psi_{i}^{*}\left(\mathbf{r}^{\prime}\right) \mathrm{e}^{-\beta \varepsilon_{i}} \psi_{i}(\mathbf{r})= \\
=C_{0}\left(\mathbf{r}, \mathbf{r}^{\prime}, \beta\right) \mathrm{e}^{-\beta U\left(\mathbf{r}, \mathbf{r}^{\prime}, \beta\right)},
\end{aligned}
$$

where the sum is over the complete spectrum including the continuum. In eq. (1), $C_{0}$ is the Bloch density of a free particle, and $U$ may be regarded as an effective $\beta$-dependent potential [10], determined by the equation :

$$
\begin{aligned}
\frac{\partial}{\partial \beta}(\beta U)=V_{\mathrm{N}}(r) & -\left(\mathbf{r}-\mathbf{r}^{\prime}\right) \cdot \nabla_{r} U+ \\
& +\frac{\hbar^{2}}{2 m} \beta \nabla_{r}^{2} U-\frac{\hbar^{2}}{2 m} \beta^{2}\left(\nabla_{r} U\right)^{2} .
\end{aligned}
$$

The ground state spatial density $\rho(\mathbf{r})$ and the energy $E$ are recovered from the diagonal part of $C$, henceforth denoted by $C(\mathbf{r}, \beta)$, by Laplace inversion with respect to $\beta$ :

$$
\begin{aligned}
\rho(\mathbf{r}) & =\mathfrak{L}_{\mu}^{-1}[C(\mathbf{r}, \beta) / \beta] \\
& \equiv \frac{1}{2 \pi i} \int_{b-i \infty}^{b+i \infty} \frac{1}{\beta} C(\mathbf{r}, \beta) \mathrm{e}^{\beta \mu} \mathrm{d} \beta, \\
E & =\int \mathrm{d}^{3} r \mathcal{L}_{\mu}^{-1}\left[-\frac{1}{\beta} \frac{\partial C(\mathbf{r}, \beta)}{\partial \beta}\right] .
\end{aligned}
$$

Here the chemical potential $\mu$ is determined by normalizing $\rho$ to the number $Z$ of electrons. So far, all the equations are exact, and we now proceed to discuss successive approximations for $U$.

It is easily seen from eq. (2) that for $\beta \rightarrow 0$,

$$
U(\mathbf{r}, \mathbf{r}, \beta) \equiv U(\mathbf{r}, \beta)=V_{\mathrm{N}}(r),
$$

which leads to the well known TF result. A better approximation [10] is obtained, however, by retaining all terms but the last in eq. (2), leading to a linear differential equation for $U$ whose solution we denote by $U_{1}$. For the Coulomb potential $V_{\mathrm{N}}$, we find

$$
\begin{aligned}
U_{1}(r, \beta)=V_{\mathrm{N}}(r)[1 & \left.-\exp \left(-\frac{r^{2}}{\hbar^{2}}\right)\right]- \\
& -\frac{Z e^{2}}{\lambda} \sqrt{\pi}\left[1-\operatorname{erf}\left(\frac{r}{\lambda}\right)\right]
\end{aligned}
$$

where $\hbar=\left(\hbar^{2} \beta / 2 m\right)^{1 / 2}$ is the thermal wave length. This result, which is exact to first order in $V_{\mathrm{N}}$, has also been obtained in a perturbative treatment [11]. Note that this effective potential $U_{1}$ is already nonsingular at the origin and varies linearly for small $r$. To our knowledge, it has not been used so far to calculate the ground state electron density in a Coulomb potential. Before proceeding to do this, we take account of the neglected nonlinear term in eq. (2) perturbatively, by substituting $U_{1}$ into the right-hand side of this equation. This leads us to the following approximate solution :

$$
\begin{aligned}
\tilde{U}(r, \beta)=U_{1}(r, \beta) & -\frac{\hbar^{2}}{8 m \beta} \times \\
& \times \int_{0}^{\beta} \beta^{\prime 2} \mathrm{~d} \beta^{\prime}\left[\frac{\mathrm{d}}{\mathrm{d} r} U_{1}\left(r, \beta^{\prime}\right)\right]^{2} .
\end{aligned}
$$

The corresponding Bloch density, $\tilde{C}(r, \beta)$, is expected to be a good approximation in the small- $\beta$ limit. The integral in eq. (6) is straightforward to evaluate. Near the origin, the effective potential $\tilde{U}(r, \beta)$ goes like

$$
\tilde{U}(r, \beta) \underset{r \rightarrow 0}{\longrightarrow}-Z e^{2}\left[\frac{\sqrt{\pi}}{\hbar}+Z e^{2} \cdot \frac{m}{2 \hbar^{2}}-\frac{r}{\hbar^{2}}+\cdots\right]
$$

whereas, its asymptotic behaviour for $r \gg \pi$ is given by

$$
\tilde{U}(r, \beta) \sim V_{\mathrm{N}}(r)-\frac{\hbar^{2}}{24 m} \beta^{2}\left[\nabla V_{\mathrm{N}}(r)\right]^{2} .
$$

This last form is exactly obtained from the semiclassical Wigner-Kirkwood expansion [12] by resumming $[13,14]$ terms containing $\nabla V$ to all orders in $\hbar$. This leads to densities which are finite at the classical turning point (for $\mu \neq 0$ ) and fall off smoothly beyond it.

The density $\tilde{\rho}(r)$ obtained in our approximation through eq. (3) is not only finite at the origin, but also satisfies the theorem [15]

$$
\rho^{\prime}(0)=-\frac{2 Z}{a_{0}} \rho(0), \quad\left(a_{0}=\frac{\hbar^{2}}{m e^{2}}\right),
$$

which holds exactly for the most general atomic system. This may be easily seen by Laplace inverting the derivative of $\tilde{C}$ at $r=0$ :

$$
\tilde{C}^{\prime}(0, \beta)=-\beta \tilde{C}(0, \beta) \tilde{U}^{\prime}(0, \beta)=-\frac{2 Z}{a_{0}} \tilde{C}(0, \beta),
$$

which follows from eq. (7). This relation will remain valid even when our method is extended to the selfconsistent atomic problem. We mention in passing that in the energy density approach with the adjusted Weizsäcker coefficient, this theorem is violated [16].

In our numerical work, the Laplace inversions in eqs. (3) and (4) are performed by the method of steepest descent, thereby evaluating the contour integrals using only the small $\beta$ behaviour of $\tilde{C}(r, \beta)$. This emphasizes the semiclassical nature of our approximation. For each integral, the real saddle point $\beta_{0}$ of the integrand is numerically found as a root of an algebraic equation, and the density $\tilde{\rho}$ is expressed analytically [17] in terms of $\widetilde{U}\left(r, \beta_{0}\right)$. As 


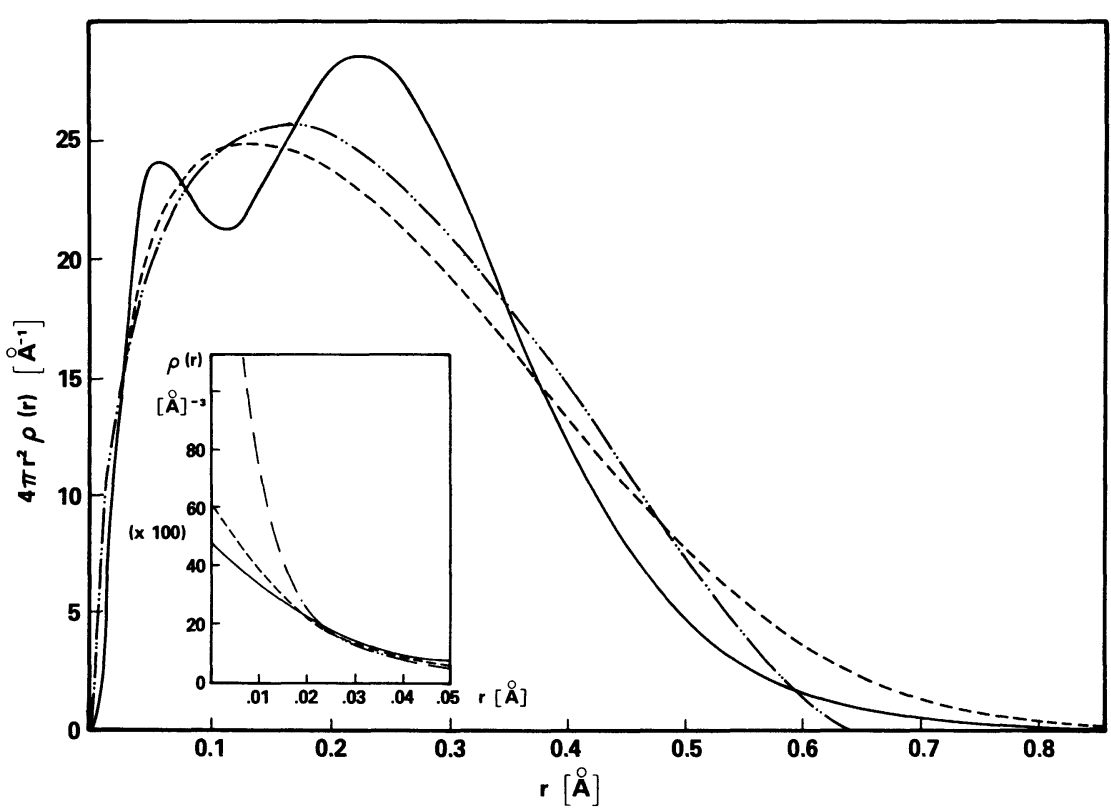

Fig. 1. - Radial density of $Z=10$ electrons in the bare Coulomb potential. Solid line : exact quantum-mechanical density. Dashed line : our present approximation. Dash-dotted line : Thomas-Fermi density.

an illustration of our method, we show in figure 1 the density $\tilde{\rho}$ in the model problem with $Z=10$ electrons and compare it with the exact quantum mechanical solution $\rho$ and the simplest Thomas-Fermi approximation $\rho_{\mathrm{TF}}$. Note the good agreement of $\tilde{\rho}$ with the exact $\rho$ for small $r$, where $\rho_{\mathrm{TF}}$ diverges (see insert in figure 1). For large $r, \tilde{\rho}$ falls off too slowly, but this is of little consequence to the energy. In figure 2, we compare the energies $E, \tilde{E}$ and $E_{\mathrm{TF}}$ as functions of $Z$. For the exact energy $E$ (solid line), each segment corresponds to the filling of a shell. The energies $\tilde{E}$ obtained in the present scheme are shown by the heavy dots. These are seen to agree very well (to within $0.3 \%$ for $Z \geqslant 0$ ) with $E$ at the magic numbers, and less accurately (better than $3 \%$ ) for midshell cases. Actually $\tilde{E}(Z)$ amounts to a smooth interpolation (dashed line) between the exact energies at the magic numbers [18].

Encouraged by these results, we now generalize our model to include the electron-electron interaction. In the mean field approximation, the hamiltonian for an electron is given by

where

$$
H=-\frac{\hbar^{2}}{2 m} \nabla^{2}-\frac{Z e^{2}}{r}+V_{\mathrm{e}}(r)
$$

$$
V_{\mathrm{e}}=e^{2} \int \mathrm{d}^{3} r^{\prime} \rho\left(\mathbf{r}^{\prime}\right) /\left|\mathbf{r}-\mathbf{r}^{\prime}\right|+V_{\mathrm{x}},
$$

the latter being the exchange potential which may be conveniently approximated by a local functional of $\rho$. The potential $V_{\mathrm{e}}$ is of course well-behaved everywhere and screens the nuclear potential $V_{\mathrm{N}}=-Z e^{2} / r$ at large distances. To include $V_{\mathrm{e}}$ in the effective potential, we write $\tilde{U}=\tilde{U}_{\mathrm{N}}+\tilde{U}_{\mathrm{e}}$, where $\tilde{U}_{\mathrm{N}}$ is given by eqs. (5) and (6). As before, $\tilde{U}$ is deter-

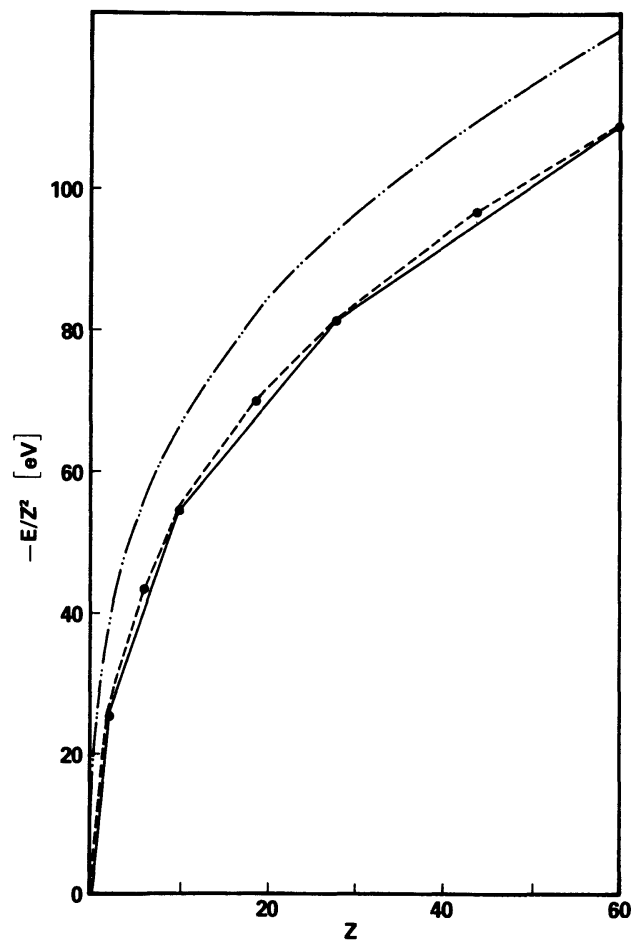

Fig. 2. - Total energy in the bare Coulomb potential versus number $Z$ of electrons. Solid line : exact sum of occupied levels. Dashed line : interpolation formula $[18] \bar{E}(Z)$. The heavy dots show our present results. Dashed-dotted lines : TF energy.

mined by eq. (2), but with $V_{\mathrm{N}}$ replaced by $V_{\mathrm{N}}+V_{\mathrm{e}}$. To leading order, $\tilde{U}_{\mathrm{e}}=V_{\mathrm{e}}$, followed by correction terms involving gradients of $\tilde{U}_{\mathrm{N}}$ and $V_{\mathrm{e}}$. In the preliminary numerical result reported here, we make the simplest approximation $\tilde{U}=\widetilde{U}_{\mathrm{N}}+V_{\mathrm{e}}+V_{\mathrm{x}}$, where the exchange potential $V_{x}$, is replaced by the Slater approximation which gives then an overall local 
potential. Starting with an initial guess of $\rho$, we calculate $\tilde{U}$ and the corresponding Bloch density $\tilde{C}(r, \beta)$. This in turn yields a new density through Laplace inversion by the steepest descent method. This procedure is repeated on a computer till self-consistency is achieved.

As an example we give in the table I our values $E$ for the binding energies of several atoms compared

Table I. - Semiquantal binding energies of several atoms compared with the exact Hartree-Fock values $E_{\mathrm{HF}}$.

$\begin{array}{ccc}Z & E(\mathrm{keV}) & E_{\mathrm{HF}}(\mathrm{keV}) \\ - & - & - \\ { }^{54} \mathrm{Xe} & -202.2 & -196.76 \\ { }^{40} \mathrm{Zr} & -99.47 & -96.30 \\ { }^{36} \mathrm{Kr} & -77.51 & -74.87 \\ { }^{20} \mathrm{Ca} & -19.23 & -18.42 \\ { }^{18} \mathrm{Ar} & -14.97 & -14.34 \\ { }^{10} \mathrm{Ne} & -3.68 & -3.50\end{array}$

with the exact Hartree-Fock values $E_{\mathrm{HF}}$. We see that the agreement with the exact results is $\sim 3 \%$. This means a considerable improvement over pure ThomasFermi theory and we believe that the accuracy of our method will still improve when higher order terms in $\tilde{U}_{\mathrm{e}}$ are included.

Our method can be extended to the multicentre molecular problem. The nuclear part of the effective potential $U_{\mathrm{N}}$ is easy to calculate since it is determined by $U_{1}$, which in turn is a linear superposition of the contribution from the individual centres.

In conclusion, our extension of the effective potential formalism of March and collaborators [10] may be an efficient tool for investigating the bulk properties of atomic and molecular systems.

This work was supported in part by the National Research Council of Canada. One of us (H. G.) acknowledges a grant by the Deutsche Forschungsgemeinschaft. Two of the authors (M. B. and P. S.) thank the members of the Physics Department of McMaster University for the warm hospitality extended to them during their visits.

\section{References}

[1] Gomвás, P., Handb. Phys., Ed. S. Flügge (Springer-Verlag) 1956, Vol. XXXVI, p. 109.

[2] Yonei, K. and Tomishima, Y., J. Phys. Soc. Japan 20 (1965) 1051

[3] Tomishima, Y. and YoneI, K., J. Phys. Soc. Japan 21 (1966) 142.

[4] YoneI, K., J. Phys. Soc. Japan 22 (1967) 1127

[5] Ashby, N. and Holzman, M. A., Phys. Rev. A 1 (1970) 764.

[6] Enstrom, J. E., Phys. Rev. A 4 (1971) 460.

[7] Baraff, G. A. and Borowitz, S., Phys. Rev. 121 (1961) 1704 ; see also Baraff, G. A., Phys. Rev. 123 (1961) 2087.

[8] Gross, E. K. U. and DreizLeR, R. M., University of Frankfürt, Preprint (1979).

[9] Thomas, L. H., Proc. Cambridge Philos. Soc. 23 (1926) 542; FERMI, E., Z. Phys. 48 (1928) 73.

[10] Hilton, D., March, N. H. and Curtis, A. R., Proc. Roy. Soc. A 300 (1967) 391;

see also March, N. H., Phys. Lett. 64A (1977) 185 and references quoted therein.

[11] Trubnikov, B. A. and Elesin, V. F., Sov. Phys. JeTP 20 (1965) 866

[12] see, e.g., Jennings, B. K., Bhaduri, R. K. and Brack, M.,
Nucl. Phys. A 253 (1975) 29, and references quoted therein.

[13] Bhaduri, R. K., Phys. Rev. Lett. 39 (1977) 329.

[14] Durand, M., Brack, M. and Schuck, P., Z. Phys. A 286 (1978) 381

[15] Steiner, E., J. Chem. Phys. 39 (1963) 2365; and Kato, T., Commun. Pure Appl. Math. 10 (1951) 151.

[16] Actually, the theorem is fulfilled when the original Weizsäcker term is used, see Goodisman, J., Phys. Rev. A 1 (1970) 1574.

[17] See Ref. [13] for details. Note that corrections, taking into account the anharmonic behaviour at the saddle point, are important for the computation of the total energy.

[18] The exact energies (in a.u.) at the magic numbers may be interpolated by $\bar{E}(Z)=-Z^{2} N(Z)$, where $N(Z)$ is the solution of $Z(N)=\frac{1}{3} N(N+1)(2 N+1)$. A very accurate approximation (to within $0.01 \%$ for $Z>1$ ) is

$$
N(Z)=\left(\frac{3 Z}{2}\right)^{1 / 3} \times\left[1+0.0630 Z^{-2 / 3}\right]-\frac{1}{2} .
$$

